\title{
Community-Based Healthcare Programs Sustainability Impact on the Sustainability of Host Organizations: A Structural Equation Modeling Analysis
}

\author{
Sebastian Ion Ceptureanu * $*$ and Eduard Gabriel Ceptureanu \\ Department of Management, The Bucharest University of Economic Studies, 010374 Bucharest, Romania; \\ eduard.ceptureanu@man.ase.ro \\ * Correspondence: sebastian.ceptureanu@man.ase.ro; Tel.: +40-7400-19879
}

Received: 13 September 2019; Accepted: 19 October 2019; Published: 21 October 2019

\begin{abstract}
The sustainability of community-based programs represents a major focus of the literature on community-based interventions in the last few decades. However, without sustainable host organizations to effectively implement them, many are prone to failure. This paper analyzes the influence of the sustainability factors of healthcare community-based programs on the host organization's sustainability. Based on a sample of 11 community-based healthcare programs and 401 respondents and using structural equation modeling, the study investigated if program specific, organization specific, and community specific factors are indeed measures of community-based programs' sustainability, if social and economic dimensions are measures of host organization sustainability, and if the sustainability of the community-based program influences thee host organization's sustainability. The results confirmed all three research hypothesis. The main contribution of the paper is to demonstrate a direct relationship between the sustainability of community-based programs and the overall sustainability of the organizations implementing them.
\end{abstract}

Keywords: community-based program; host organization; sustainability; Structural Equation Modeling

\section{Introduction}

Community-based programs ( $\mathrm{CbPs})$ have been increasingly present in the literature in the last few decades [1-4] due to their impact on local communities such as through an efficient, transparent, and more equitable use of local resources [5-8] or improving the standards of living in the targeted communities [9-12].

The sustainability of $\mathrm{CbPs}$ is also important for the host organization, since it may also affect their sustainability [13]. An important share of the CbPs terminates unexpectedly in their very first few years after initiation, usually after the initial support is finished [1], thus failing to provide significant results for the communities. Unexpected termination of CbPs leads to negative effects for the targeted communities, since they may lose trust in similar such initiatives in the future, but also for the host organizations, since the initiation costs for a community-based program are usually high [14]. However, sustainability is rarely included in $\mathrm{CbP}$ planning [15], with items like program immediate outcomes prevailing instead [16-18].

Various studies have analyzed $\mathrm{CbP}$ sustainability by focusing on its continuation after a targeted intervention has been terminated [1,3-5]. In this case, activities are implemented according to the program goals $[18,19]$. Others have argued that CbPs may be considered sustainable only after their institutionalization in relevant organizations [20,21].

While the literature presenting the sustainability of community-based programs and organizational sustainability is extensive, the relationship between $\mathrm{CbP}$ sustainability and sustainability of the host 
organization has rarely been analyzed. This constitutes the rationale of this study and the research gap that it plans to fill.

The rest of this paper is organized as follows. Section 1 provides the rationale of the study and presents the research problem. Section 2 presents the literature review divided into two subsections, each one providing the theoretical background of $C b P$ sustainability and Host organization sustainability, the research hypothesis and the variables for each construct. Section 3 presents the material and methods employed in analyzing the research problem. Section 4 present the results and discussion. Section 5 discusses the implications of the findings and highlights our conclusions.

\section{Literature Review}

\subsection{Community-Based Program Sustainability}

Community-based programs represent social interventions seeking to change social structures and institutions in the community. Their sustainability is inconsistently defined in the literature [22], especially due to the diverse landscape of CbPs and the multitude of stakeholders involved in their planning, implementation, and assessment [10]. Community-based program sustainability is considered in the literature in terms of the program continuing in its entirety [1,2], the continuity of specific program components [22], the improvement of community capacity $[13,20,21]$, or the capacity of the $\mathrm{CbP}$ to continuously respond to community problems [23], which is proof of the heterogeneity of existing approaches.

CbPs intrinsically rely on community-based approaches by creating partnerships throughout their implementation [4]. Therefore, their sustainability depends not only on themselves and the capabilities of the host organizations to implement them, but also on the stakeholders [24]. Moreover, CbPs not only require the targeted community's acceptance, but also community involvement. Thus, to be successful in the long term, CbPs have to incorporate the targeted community's needs and accept stakeholder involvement [25] by improving program accountability [26]. As CbPs must consider and account for the targeted communities' cultural identities to be sustainable [27], without socio-cultural acceptability, their chances of being sustainable will be low, hindered during implementation by a lack of trust or rejection [3].

Unfortunately, a comprehensive overview of the CbP sustainability factors does not exist [23,28,29]. Various investigations have used different and sometimes divergent approaches to identify different $\mathrm{CbPs}$ sustainability factors, therefore making it difficult to assess the relevance and relative importance of each one. There are general approaches, emphasizing broader categories of factors such as the importance of key people involved in program implementation [13,30,31], the organizational setting of the host organization [11,13,14,30-33], the social and political environment of the community-based program $[11,13,17,32,33]$, and the financial resources required or available for program implementation [11,14,32]. Other authors [34-50] have argued that CbP sustainability is determined by specific factors such as program champions, stakeholder capabilities, program leadership, effective collaboration with the targeted community, or community support for the CbP.

This study used a previously tested model [4] comprising of three main factors: (1) related to the program itself; (2) related to the host organization, and (3) related to the community. The model was proven to be suitable for the Romanian context [4] (Table 1). 
Table 1. Community-based program sustainability construct.

\begin{tabular}{|c|c|c|}
\hline Variables & Description & References \\
\hline \multicolumn{3}{|r|}{ I. Program Specific } \\
\hline $\begin{array}{l}\text { Program coordinator } \\
\text { competence }\end{array}$ & $\mathrm{CbP}$ coordinator ability effectively run the program & Akerlund, 2000; Hanson \& Salmoni, 2011; Montemurro et al., 2014; Mancini \& Marek, 2004; Ceptureanu et al., 2018 \\
\hline Program transparency & $\begin{array}{l}\text { CbP capability to inform stakeholders of its results } \\
\text { and outcomes, using suitable methods }\end{array}$ & O’Loughlin et al., 1998; Holder \& Moore, 2000; Savaya et al., 2008; Ceptureanu et al., 2018 \\
\hline $\begin{array}{l}\text { Qualified HR } \\
\text { involvement }\end{array}$ & use of qualified staff in all stages of $\mathrm{CbP}$ & $\begin{array}{l}\text { O’Loughlin et al., 1998; Holder \& Moore, 2000; Mancini \& Marek, 2004; Estabrooks et al., 2011; Hanson \& Salmoni, 2011; } \\
\text { Ceptureanu et al., } 2018\end{array}$ \\
\hline Program responsivity & $\mathrm{CbP}$ ability to address changes of community needs & Akerlund, 2000; Holder \& Moore, 2000; Mancini \& Marek, 2004; Ceptureanu et al., 2018 \\
\hline Program funding & $\mathrm{CbP}$ availability of financial resources & $\begin{array}{l}\text { Light, 1998; Shediac-Rizkallah \& Bone, 1998; Akerlund, 2000; Holder \& Moore, 2000; Goodson et al., 2001; Steadman et al., } \\
\text { 2002; Mancini \& Marek, 2004; Scheirer, 2005; Stevens \& Peikes, 2006; Estabrooks et al., 2011; Oino et al., 2015; } \\
\text { Ceptureanu et al., } 2018\end{array}$ \\
\hline Program theory & CbP coherent framework & Steadman et al., 2002; Weiss et al., 2002; Savaya et al., 2008; Ceptureanu et al., 2018 \\
\hline Program effectiveness & $\begin{array}{l}\text { CbP capability to document its success and make it } \\
\text { visible for stakeholders }\end{array}$ & $\begin{array}{l}\text { Shediac-Rizkallah \& Bone, 1998; Pentz, 2000; Steadman et al., 2002; Mancini \& Marek, 2004; Padgett et al., 2005; } \\
\text { Ceptureanu et al., } 2018\end{array}$ \\
\hline Program flexibility & $\begin{array}{l}\mathrm{CbP} \text { ability to adapt and evolve from the original } \\
\text { plan, according to changing circumstances }\end{array}$ & O'Loughlin et al., 1998; Scheirer, 2005; Savaya et al., 2008; Ceptureanu et al., 2018 \\
\hline Program evaluation & $\begin{array}{l}\mathrm{CbP} \text { capability to align to the reporting } \\
\text { requirements of stakeholders }\end{array}$ & Weiss et al., 2002; Johnson et al., 2004; Savaya et al., 2008; Ceptureanu et al., 2018 \\
\hline Program champions & $\begin{array}{l}\text { individuals related to } \mathrm{CbP} \text { promoting it in the } \\
\text { community }\end{array}$ & $\begin{array}{l}\text { Smith et al., 1993; Shediac-Rizkallah \& Bone, 1998; O'Loughlin et al., 1998; Holder \& Moore, 2000; Goodson et al., 2001; } \\
\text { Steadman et al., 2002; Mancini \& Marek, 2004; Scheirer, 2005; Savaya et al., 2008; Ceptureanu et al., } 2018\end{array}$ \\
\hline Program integration & $\begin{array}{l}\text { CbP of dependence to the host organization in terms } \\
\text { of mission and strategy }\end{array}$ & $\begin{array}{l}\text { Smith et al., 1993; Shediac-Rizkallah \& Bone, 1998; O'Loughlin et al., 1998; Goodson et al., 2001; Johnson et al., 2004; } \\
\text { Padgett et al., 2005; Ceptureanu et al., } 2018\end{array}$ \\
\hline $\begin{array}{l}\text { Understanding the } \\
\text { community }\end{array}$ & $\begin{array}{l}\mathrm{CbP} \text { capability to identify and integrate community } \\
\text { needs and resources }\end{array}$ & $\begin{array}{l}\text { Shediac-Rizkallah \& Bone, 1998; Holder \& Moore, 2000; Pentz, 2000; Mancini et al., 2003; Mancini \& Marek, 2004; } \\
\text { Oino et al., 2015; Ceptureanu et al., } 2018\end{array}$ \\
\hline Political legitimation & $\begin{array}{l}\mathrm{CbP} \text { adaptation to the policies and regulations of } \\
\text { relevant stakeholders }\end{array}$ & Pentz, 2000; Pluye et al., 2004; Sarriot et al., 2004; Scheirer, 2005; Ceptureanu et al., 2018 \\
\hline \multicolumn{3}{|r|}{ II. Organizational Specific } \\
\hline Leadership & $\begin{array}{l}\text { host organization senior management capacity to } \\
\text { establish organizational goals congruent with } \mathrm{CbP}\end{array}$ & $\begin{array}{l}\text { Shediac-Rizkallah \& Bone, 1998; Akerlund, 2000; LaFond et al., 2002; Sarriot et al., 2004; Mancini \& Marek, 2004; } \\
\text { Nu'Man et al., 2007; Jacobs et al., 2007; Argaw et al., 2007; Gruen et al., 2008; Ceptureanu et al., } 2018\end{array}$ \\
\hline Organizational system & $\begin{array}{l}\text { host organization procedures and mechanisms (HR } \\
\text { and financing), which may impact } \mathrm{CbP} \text { outcomes }\end{array}$ & $\begin{array}{l}\text { Shediac-Rizkallah \& Bone, 1998; LaFond et al., 2002; Mancini \& Marek, 2004; Sarriot et al., 2004; Johnson et al., 2004; } \\
\text { Beery et al., 2005; Robinson et al., 2005; Jacobs et al., 2007; Nu'Man et al., 2007; Gruen et al., 2008; Estabrooks et al., 2011; } \\
\text { Mijnarends et al., 2011; Ceptureanu et al., 2018; }\end{array}$ \\
\hline Organizational stability & $\begin{array}{l}\text { host organization ability to adapt its internal } \\
\text { regulations and procedures, which may impact } \mathrm{CbP} \\
\text { outcomes }\end{array}$ & $\begin{array}{l}\text { Shediac-Rizkallah \& Bone, 1998; Goodson et al., 2001; LaFond et al., 2002; Sarriot et al., 2004; Johnson et al., 2004; } \\
\text { Pluye et al., 2005; Argaw et al., 2007; Nu'Man et al., 2007; Jacobs et al., 2007; Gruen et al., 2008; Ceptureanu et al., 2018; }\end{array}$ \\
\hline
\end{tabular}


Table 1. Cont.

\begin{tabular}{|c|c|c|}
\hline Variables & Description & References \\
\hline Partnering & $\begin{array}{l}\text { host organization capacity to initiate and maintain } \\
\text { relations with multiple partners, which may impact } \\
\text { CbP outcomes }\end{array}$ & $\begin{array}{l}\text { LaFond et al., 2002; Sarriot et al., 2004; Nu'Man et al., 2007; Hanson \& Salmoni, 2011; Montemurro et al., 2014; Oino et al., } \\
\text { 2015; Ceptureanu et al., } 2018\end{array}$ \\
\hline $\begin{array}{l}\text { Specific sustainability } \\
\text { actions }\end{array}$ & $\begin{array}{l}\text { host organization actions specifically targeting } \\
\text { sustainability, which may impact CbP outcomes }\end{array}$ & Johnson et al., 2004; Beery et al., 2005; Robinson et al., 2005; Ceptureanu et al., 2018 \\
\hline \multicolumn{3}{|r|}{ III. Community Specific } \\
\hline $\begin{array}{l}\text { Community } \\
\text { participation }\end{array}$ & $\begin{array}{l}\text { targeted community involvement in } \mathrm{CbP} \text { planning } \\
\text { and implementation }\end{array}$ & $\begin{array}{l}\text { Sarriot et al., 2004; Sarriot et al., 2004; Jacobs et al., 2007; Argaw et al., 2007; Gruen et al., 2008; Oino et al., 2015; } \\
\text { Ceptureanu et al., } 2018\end{array}$ \\
\hline $\begin{array}{l}\text { Community political } \\
\text { context }\end{array}$ & $\begin{array}{l}\text { targeted community relations with various public or } \\
\text { private bodies and agencies, which may impact } \mathrm{CbP} \\
\text { outcomes }\end{array}$ & $\begin{array}{l}\text { Shediac-Rizkallah \& Bone, 1998; Weiss et al., 2002; Sarriot et al., 2004; Sarriot et al., 2004; Jacobs et al., 2007; Gruen et al., } \\
\text { 2008; Estabrooks et al., 2011; Mijnarends et al., 2011; Ceptureanu et al., } 2018\end{array}$ \\
\hline Community support & $\begin{array}{l}\text { targeted community involvement in providing } \\
\text { additional resources to CbP, particularly financial } \\
\text { contributions }\end{array}$ & Sarriot et al., 2004; Sarriot et al., 2004; Jacobs et al., 2007; Gruen et al., 2008; Montemurro et al., 2014; Ceptureanu et al., 2018 \\
\hline Community capacity & $\begin{array}{l}\text { target group }(\mathrm{s}) \text { availability for } \mathrm{CbP} \text { from targeted } \\
\text { community }\end{array}$ & $\begin{array}{l}\text { Sarriot et al., 2004; Sarriot et al., 2004; Jacobs et al., 2007; Gruen et al., 2008; Hanson \& Salmoni, 2011; Hacker et al., 2012; } \\
\text { Montemurro et al., 2014; Oino et al., 2015; Ceptureanu et al., } 2018\end{array}$ \\
\hline
\end{tabular}


Therefore, we hypothesized that

Hypothesis 1. Program specific, organizational specific, and community specific constructs are measures of CbP sustainability.

\subsection{Host Organization Sustainability}

We considered the sustainability of non-profits in discussing organizational sustainability. This ensures a comprehensive range for organizations that may act as host organizations for community-based programs. Sustainability for non-profits means that they continue to fulfill their mission and satisfy the key stakeholders' requirements, regardless of the difficulties encountered [51].

For non-profits, the donors assume a central role because they are critical actors in providing the revenue flow [51]. Non-profits also have to cope with the significant volatility of their revenue sources, making multiple stakeholder management more complex. More stakeholders mean that non-profits have to find a fine balance between money and their mission [52,53]. On the other hand, non-profits have a broader range of mechanisms to ensure a flow of resources for support [54] by acquiring funds through governmental support and private donations, commercially generated revenues, fundraising and donations, cross sector partnerships, or volunteerism [55-61].

There are different perspectives on the sustainability of non-profits. One approach focuses on the financial viability [62-68], probably the most common in the literature and an important factor in considering the economic dimension in our model.

Another approach, focused on organization [69], still considers funding as important, but has a more balanced approach, bringing forward items like leadership, program development and management, or the quality of resources. A development of this approach $[70,71]$ links non-profit sustainability to several factors, namely strategy, culture, operations, people, and the business model.

In parallel with these approaches, the social mission of non-profits is discussed in the literature in relation with sustainability, since many argue that the ultimate goal of non-profits is to increase social value [72-75]. This provided the rationale for part of the second dimension of the Host organization sustainability factor, the social one.

Finally, one last approach, a result of increased marketization of the non-profit sector, focuses on the implementation of business principles in non-profits [76]. To survive, non-profits are becoming more entrepreneurial [77], become more adept with innovative practices and improve attitude toward change [53], or begin redefining their mission [78,79]. This provided the rationale for the second dimension of the model, namely, the social dimension.

Again, for host organization sustainability construct, we used a previous tested model [80], comprising Social Dimension with the items Mission achievement, Public image, Risk acceptance, Initiative, Attitude toward change and Entrepreneurial approach, and Economic Dimension with the items Reporting compliance, Revenue diversification, Financial planning and Stability of revenue [80-92] (Table 2): 
Table 2. Host organization sustainability construct.

\begin{tabular}{|c|c|c|}
\hline \multicolumn{3}{|r|}{ I. Social Dimension } \\
\hline Mission achievement & $\begin{array}{l}\text { Host organization degree of achievement of its } \\
\text { mission }\end{array}$ & Shediac-Rizkallah \& Bone, 1998; Prahalad, 2004; Gray \& Stites, 2013; Ceptureanu et al., 2017 \\
\hline Public image & Host organization image for stakeholders & Helmig et al., 2004; Jegers \& Lapsley, 2004; Ceptureanu et al., 2017; Ceptureanu et al., 2018 \\
\hline Risk acceptance & Host organization willingness to accept risks & $\begin{array}{l}\text { Thompson et al., 2000; Alvord et al., 2004; Turner \& Martin, 2005; Peredo \& McLean, 2006; Mair \& Marti, 2006; Nicholls, } \\
\text { 2006; Austin et al., 2006; Ceptureanu et al., } 2017\end{array}$ \\
\hline Initiative & $\begin{array}{l}\text { Host organization availability to get involved in } \\
\text { new activities and initiatives }\end{array}$ & Sharir \& Lerner, 2006; Nicholls, 2006; Ceptureanu et al., 2017 \\
\hline Attitude toward change & $\begin{array}{l}\text { Host organization willingness to implement new } \\
\text { processes and procedures }\end{array}$ & Alvord et al., 2004; Parsons \& Broadbridge, 2004; Ceptureanu et al., 2017 \\
\hline $\begin{array}{l}\text { Entrepreneurial } \\
\text { approach }\end{array}$ & $\begin{array}{l}\text { Host organization availability to target new } \\
\text { beneficiaries }\end{array}$ & Turner \& Martin, 2005; Austin et al., 2006; Iwu et al., 2015; Ceptureanu et al., 2017 \\
\hline \multicolumn{3}{|r|}{ II. Economic Dimension } \\
\hline Reporting compliance & $\begin{array}{l}\text { Host organization compliance with specific } \\
\text { stakeholders rules and requirements in terms of } \\
\text { reporting }\end{array}$ & Zietlow et al., 2007; McLaughlin, 2009; Coe, 2011; Murtaza, 2012; Weikart et al., 2013; Prentice, 2016; Ceptureanu et al., 2017 \\
\hline Revenue diversification & Host organization number of sources of revenue & Greenlee \& Trussel, 2000; Keating et al., 2005; Hodge \& Piccolo, 2005; Prentice, 2016; Ceptureanu et al., 2017 \\
\hline Financial planning & $\begin{array}{l}\text { Host organization capability to implement adequate } \\
\text { financial planning }\end{array}$ & $\begin{array}{l}\text { Keating et al., 2005; Zietlow et al., 2007; McLaughlin, 2009; Coe, 2011; Weikart et al., 2013; Prentice, 2016; Ceptureanu et al., } \\
2017\end{array}$ \\
\hline Stability of revenue & Host organization financial result perspective & Hodge \& Piccolo, 2005; Keating et al., 2005; Zietlow et al., 2007; McLaughlin, 2009; Prentice, 2016; Ceptureanu et al., 2017 \\
\hline
\end{tabular}


Therefore, we hypothesized that

Hypothesis 2. Social dimension and economic dimension are measures of organizational sustainability.

In the end, one more assumption was made:

Hypothesis 3. CbP sustainability positively and significantly influences host organization sustainability.

\section{Materials and Methods}

For sampling, a purposive sampling strategy was used. Homoscedasticity was checked through Levene's test [93]. Tests on the homogeneity of variances indicted that the sample across all of the control variables were homogeneous (indicated by Levene statistic $>0.05$ ) on all control variables: host organization target (urban/rural), age, and size. The respondents were associated with specific community-based healthcare programs. Thus, $\mathrm{N}_{1}\left(\mathrm{~N}_{1}=11\right)$ represents the number of host organizations surveyed, each one implementing one community-based healthcare program, while $\mathrm{N}_{2}$ represents the number of respondents associated with a specific healthcare CbP. These include members of the community, employees, and volunteers of host organizations engaged in community-based program implementation. No respondents were allowed to provide answers for more than one $\mathrm{CbP}$. The sample composition is presented in Table 3.

Table 3. Sample composition.

\begin{tabular}{|c|c|c|c|}
\hline Criteria & Description & $\begin{array}{l}\text { Number of Host } \\
\text { Organizations } \\
\left(\mathrm{N}_{1}=11\right)\end{array}$ & $\begin{array}{c}\text { Associated } \\
\text { Respondents } \\
\left(\mathrm{N}_{2}=401\right)\end{array}$ \\
\hline \multirow{2}{*}{ Host organization target (area of operations) } & urban & 7 & 257 \\
\hline & rural & 4 & 144 \\
\hline \multirow{2}{*}{ Host organization age (no. of years since establishment) } & $<5$ & 3 & 112 \\
\hline & $>5$ & 8 & 289 \\
\hline \multirow{2}{*}{ Host organization size (no. of employees, excluding volunteers) } & $<10$ & 2 & 330 \\
\hline & $>10$ & 9 & 71 \\
\hline \multirow{3}{*}{ Type of support } & Non-profit support & 2 & 43 \\
\hline & Local support & 6 & 96 \\
\hline & County support & 3 & 262 \\
\hline \multirow{5}{*}{ Target } & $\begin{array}{l}\text { Smoking } \\
\text { prevention }\end{array}$ & 1 & 27 \\
\hline & Diabetes & 2 & 52 \\
\hline & $\begin{array}{l}\text { Heart diseases } \\
\text { prevention }\end{array}$ & 2 & 83 \\
\hline & Mixed & 5 & 203 \\
\hline & Healthy nutrition & 1 & 36 \\
\hline
\end{tabular}

Most of the host organizations operating in urban areas (64\%) had more than five years of expertise in implementing community-based programs (73\%) and were large (82\%). In terms of support provided, two CbPs were supported by non-profits, while most were supported by town hall or county authorities. Healthcare services included heart disease prevention and diabetes (equally $18 \%$ ), smoking prevention $(9 \%)$, healthy nutrition $(9 \%)$, and mixed $(46 \%)$. The questionnaires were collected over a period of eight months, from February to September 2019. No ethical issues arose during data collection, while the respondents were assured about the confidentiality of their answers. In terms of permits, since the questionnaire included only non-medical topics, no permits were necessary or required.

Exploratory factor analysis (EFA) followed by confirmatory factor analysis (CFA) were used for validation [92,93]. Factors with eigenvalues greater than 1 were retained [94,95]. Items with factor loadings larger than 0.40 were retained [96]. For EFA with individual factors, the identified number 
of items had an overall Kaiser-Meyer-Olkin (KMO) value above 7.5 [97]. The conceptual mode and research hypothesis were tested using SEM-Amos (SPSS, version 22) [98]. Orthogonal varimax with Kaiser normalization was used on all items. Path analysis further supported the findings established through EFA [99]. Regarding multicollinearity, to rule it out, variance inflationary factor (VIF) scores were checked. Table 4 shows the results with all items below the recommended threshold of $2.5[93,100]$.

Table 4. Multicollinearity results.

\begin{tabular}{|c|c|c|c|c|c|c|c|c|}
\hline \multicolumn{9}{|c|}{ Coefficients } \\
\hline & \multirow[t]{2}{*}{ Model } & \multicolumn{2}{|c|}{$\begin{array}{c}\text { Unstandardized } \\
\text { Coefficients }\end{array}$} & \multirow{2}{*}{$\begin{array}{l}\text { Standardized } \\
\text { Coefficients }\end{array}$} & \multirow[t]{2}{*}{$\mathbf{t}$} & \multirow[t]{2}{*}{ Sig. } & \multicolumn{2}{|c|}{$\begin{array}{l}\text { Collinearity } \\
\text { Statistics }\end{array}$} \\
\hline & & B & Std. Error & & & & Tolerance & VIF \\
\hline 1 & (Constant) & -0.298 & 0.788 & -0.378 & 0.706 & & & \\
\hline & \multicolumn{8}{|c|}{ Program Specific } \\
\hline & Coordinator competence & 0.269 & 0.052 & 0.259 & 5.190 & 0.000 & 0.675 & 1.481 \\
\hline & Transparency & 0.210 & 0.056 & 0.195 & 3.754 & 0.000 & 0.626 & 1.597 \\
\hline & Qualified HR involvement & 0.208 & 0.050 & 0.206 & 4.152 & 0.000 & 0.682 & 1.466 \\
\hline & Responsivity & 0.144 & 0.054 & 0.130 & 2.658 & 0.008 & 0.708 & 1.412 \\
\hline & Program funding & 0.025 & 0.055 & 0.022 & 0.455 & 0.650 & 0.687 & 1.455 \\
\hline & Program theory & 0.115 & 0.056 & 0.107 & 2.070 & 0.039 & 0.625 & 1.600 \\
\hline & Program effectiveness & -0.068 & 0.056 & -0.068 & -1.206 & 0.228 & 0.534 & 1.874 \\
\hline & Program flexibility & 0.012 & 0.054 & 0.012 & 0.230 & 0.818 & 0.657 & 1.523 \\
\hline & Program evaluation & 0.102 & 0.056 & 0.091 & 1.832 & 0.068 & 0.677 & 1.478 \\
\hline & Program champions & 0.026 & 0.055 & 0.024 & 0.479 & 0.632 & 0.663 & 1.508 \\
\hline & Program integration with the host organization & -0.077 & 0.065 & -0.057 & -1.185 & 0.237 & 0.720 & 1.389 \\
\hline & Understanding the community & 0.181 & 0.060 & 0.156 & 3.045 & 0.003 & 0.642 & 1.558 \\
\hline & Political legitimation & -0.006 & 0.055 & -0.006 & -0.115 & 0.908 & 0.662 & 1.512 \\
\hline & \multicolumn{8}{|c|}{ Organizational Specific } \\
\hline & Leadership & -0.036 & 0.065 & -0.028 & -0.548 & 0.584 & 0.624 & 1.603 \\
\hline & Organizational system & -0.164 & 0.062 & -0.136 & -2.647 & 0.008 & 0.639 & 1.564 \\
\hline & Organizational stability & 0.085 & 0.060 & 0.078 & 1.410 & 0.159 & 0.550 & 1.819 \\
\hline & Partnering & 0.093 & 0.054 & 0.085 & 1.726 & 0.085 & 0.696 & 1.437 \\
\hline & Specific sustainability actions & 0.017 & 0.056 & 0.016 & 0.300 & 0.764 & 0.601 & 1.663 \\
\hline & \multicolumn{8}{|c|}{ Community Specific } \\
\hline & Community participation & -0.005 & 0.062 & -0.004 & -0.084 & 0.933 & 0.624 & 1.604 \\
\hline & Community political context & 0.025 & 0.069 & 0.021 & 0.361 & 0.718 & 0.512 & 1.955 \\
\hline & Community support & 0.032 & 0.065 & 0.027 & 0.495 & 0.621 & 0.575 & 1.740 \\
\hline & Community capacity & -0.052 & 0.063 & -0.044 & -0.823 & 0.411 & 0.599 & 1.671 \\
\hline & \multicolumn{8}{|c|}{ Social Dimension } \\
\hline & Address social needs & -0.033 & 0.067 & -0.031 & -0.493 & 0.622 & 0.414 & 2.413 \\
\hline & Public image & 0.051 & 0.093 & 0.039 & 0.550 & 0.583 & 0.331 & 2.019 \\
\hline & Risk acceptance & 0.023 & 0.096 & 0.015 & 0.237 & 0.813 & 0.406 & 2.462 \\
\hline & Initiative & -0.082 & 0.091 & -0.057 & -0.900 & 0.369 & 0.426 & 2.346 \\
\hline & Attitude toward change & 0.041 & 0.083 & 0.031 & 0.495 & 0.621 & 0.441 & 2.266 \\
\hline & Entrepreneurial approach & -0.027 & 0.072 & -0.021 & -0.383 & 0.702 & 0.564 & 1.773 \\
\hline & \multicolumn{8}{|c|}{ Economic Dimension } \\
\hline & Reporting compliance & -0.134 & 0.068 & -0.107 & -1.963 & 0.050 & 0.572 & 1.749 \\
\hline & Revenue diversification & 0.004 & 0.057 & 0.003 & 0.066 & 0.948 & 0.873 & 1.146 \\
\hline & Financial planning & -0.143 & 0.075 & -0.113 & -1.901 & 0.058 & 0.477 & 2.095 \\
\hline & Stability of revenue & 0.093 & 0.086 & 0.074 & 1.076 & 0.283 & 0.354 & 2.821 \\
\hline
\end{tabular}

In terms of reliability, Cronbach's alpha was used. The reliability test of the data for the structure of both CbP Sustainability and Host organization sustainability factors showed good internal consistency, higher the recommended threshold of 0.7 [101] (Tables 5 and 6). 
Table 5. Reliability of CbP sustainability factors.

\begin{tabular}{cc}
\hline Factors & Cronbach's Alpha \\
\hline Program specific & 0.782 \\
\hline Organizational specific & 0.729 \\
\hline Community specific & 0.716 \\
\hline
\end{tabular}

Table 6. Reliability of Host organization sustainability factors.

\begin{tabular}{cc}
\hline Factors & Cronbach's Alpha \\
\hline Social dimension & 0.841 \\
\hline Economic dimension & 0.795 \\
\hline
\end{tabular}

EFA was used to measure the shared variance of factors and to identify the relationships between items [102]. No prior assumptions were made about the relationships between the factors. The items with eigenvalues $>1$ were considered during the EFA, while the minimum threshold of 0.4 was taken to retain the items loading on to their respective factors. After EFA, principal component analysis (PCA) with varimax rotation was performed. Table 7 shows the rotated component matrix for all of the items retained. Considering the threshold value, the loadings indicate that the factor structure is valid.

Table 7. Rotated component matrix.

\begin{tabular}{|c|c|c|c|c|c|}
\hline \multicolumn{6}{|c|}{ Rotated Component Matrix ${ }^{a}$} \\
\hline & \multicolumn{5}{|c|}{ Component } \\
\hline & 1 & 2 & 3 & 4 & 5 \\
\hline Coordinator competence & 0.858 & & & & \\
\hline Transparency & 0.747 & & & & \\
\hline Qualified HR involvement & 0.701 & & & & \\
\hline Responsivity & 0.676 & & & & \\
\hline Program funding & 0.659 & & & & \\
\hline Program theory & 0.648 & & & & \\
\hline Program effectiveness & 0.726 & & & & \\
\hline Program flexibility & 0.731 & & & & \\
\hline Program evaluation & 0.717 & & & & \\
\hline Program champions & 0.628 & & & & \\
\hline Program integration with the host organization & 0.696 & & & & \\
\hline Understanding the community & 0.702 & & & & \\
\hline Political legitimation & 0.598 & & & & \\
\hline Leadership & & 0.778 & & & \\
\hline Organizational system & & 0.716 & & & \\
\hline Organizational stability & & 0.693 & & & \\
\hline Partnering & & 0.702 & & & \\
\hline Specific sustainability actions & & 0.684 & & & \\
\hline Community participation & & & 0.626 & & \\
\hline Community political context & & & 0.611 & & \\
\hline Community support & & & 0.704 & & \\
\hline Community capacity & & & 0.722 & & \\
\hline Address social needs & & & & 0.763 & \\
\hline Public image & & & & 0.845 & \\
\hline Risk acceptance & & & & 0.715 & \\
\hline Initiative & & & & 0.607 & \\
\hline Attitude toward change & & & & 0.622 & \\
\hline Entrepreneurial approach & & & & 0.659 & \\
\hline Reporting compliance & & & & & 0.834 \\
\hline Revenue diversification & & & & & 0.817 \\
\hline Financial planning & & & & & 0.793 \\
\hline Stability of revenue & & & & & 0.784 \\
\hline
\end{tabular}

Extraction method: Principal component analysis. Rotation method: Varimax with Kaiser normalization. ${ }^{a}$ Rotation converged in six iterations. 
To analyze the relationship between the variables, the Pearson product moment correlation test was used. All correlation values showed positive correlations (Table 8).

Table 8. Correlation matrix.

\begin{tabular}{lccccc}
\hline & $\begin{array}{c}\text { Program } \\
\text { Specific }\end{array}$ & $\begin{array}{c}\text { Organizational } \\
\text { Specific }\end{array}$ & $\begin{array}{c}\text { Community } \\
\text { Specific }\end{array}$ & $\begin{array}{c}\text { Social } \\
\text { Dimension }\end{array}$ & $\begin{array}{c}\text { Economic } \\
\text { Dimension }\end{array}$ \\
\hline Program specific & 1 & & & & \\
\hline Organizational specific & $0.207^{*}$ & 1 & 1 & & \\
\hline Community specific & 0.033 & 0.042 & 0.026 & 1 & 1 \\
\hline Social dimension & $0.451^{* *}$ & 0.052 & 0.013 & $0.465^{* *}$ & 1 \\
\hline Economic dimension & $0.409^{* *}$ & 0.089 & 0.013 & \\
\hline
\end{tabular}

\section{Results and Discussion}

The SEM model shows that all of the factors were significant (Table 9). All three of the CbP Sustainability factors constructed (program specific, organizational specific, and community specific) $(p<0.001)$ were found to be significant measures of CbP Sustainability. Among the individual factors, the program specific construct was a significant measure of CbP Sustainability with a high and positive coefficient value of 0.703 , followed by organizational specific with a coefficient value of 0.682 , and community specific with a coefficient value of 0.533 . Therefore,

Hypothesis 1. Program specific, organizational specific, and community specific constructs are measures of CbP Sustainability.

is supported.

Table 9. Standardized regression weights: (complete Structural Equation Modeling model).

\begin{tabular}{|c|c|c|c|c|}
\hline & & & Estimate & $p$ Values \\
\hline Program specific & $<-$ & CbP Sustainability & 0.703 & $* * *$ \\
\hline Organizational specific & $<-$ & CbP Sustainability & 0.682 & $* * *$ \\
\hline Community specific & $<-$ & CbP Sustainability & 0.533 & $* * *$ \\
\hline Social dimension & $<-$ & Host organization sustainability & 0.368 & $* * *$ \\
\hline Economic dimension & $<-$ & Host organization sustainability & 0.612 & $* * *$ \\
\hline Host organization sustainability & $<-$ & CbP Sustainability & 0.742 & $* * *$ \\
\hline Coordinator competence & $<-$ & Program specific & 0.412 & 0.004 \\
\hline Transparency & $<-$ & Program specific & 0.674 & $* * *$ \\
\hline Qualified HR involvement & $<-$ & Program specific & 0.652 & $* * *$ \\
\hline Responsivity & $<-$ & Program specific & 0.625 & $* * *$ \\
\hline Program funding & $<-$ & Program specific & 0.599 & $* * *$ \\
\hline Program theory & $<-$ & Program specific & 0.552 & $* * *$ \\
\hline Program effectiveness & $<-$ & Program specific & 0.605 & $* * *$ \\
\hline Program flexibility & $<-$ & Program specific & 0.560 & $* * *$ \\
\hline Program evaluation & $<-$ & Program specific & 0.602 & $* * *$ \\
\hline Program champions & $<-$ & Program specific & 0.441 & 0.003 \\
\hline Program integration with the host organization & $<-$ & Program specific & 0.550 & $* * *$ \\
\hline Understanding the community & $<-$ & Program specific & 0.642 & $* * *$ \\
\hline Political legitimation & $<-$ & Program specific & 0.575 & $* * *$ \\
\hline Leadership & $<-$ & Organizational specific & 0.516 & $* * *$ \\
\hline
\end{tabular}


Table 9. Cont.

\begin{tabular}{|c|c|c|c|c|}
\hline & & & Estimate & $p$ Values \\
\hline Organizational system & $<-$ & Organizational specific & 0.715 & $* * *$ \\
\hline Organizational stability & $<-$ & Organizational specific & 0.525 & $* * *$ \\
\hline Partnering & $<-$ & Organizational specific & 0.632 & $* * *$ \\
\hline Specific sustainability actions & $<-$ & Organizational specific & 0.490 & $* * *$ \\
\hline Community participation & $<-$ & Community specific & 0.602 & $* * *$ \\
\hline Community political context & $<-$ & Community specific & 0.761 & $* * *$ \\
\hline Community support & $<-$ & Community specific & 0.667 & $* * *$ \\
\hline Community capacity & $<-$ & Community specific & 0.640 & $* * *$ \\
\hline Address social needs & $<-$ & Social dimension & 0.730 & $* * *$ \\
\hline Public image & $<-$ & Social dimension & 0.870 & $* * *$ \\
\hline Risk acceptance & $<-$ & Social dimension & 0.740 & $* * *$ \\
\hline Initiative & $<-$ & Social dimension & 0.501 & 0.001 \\
\hline Attitude toward change & $<-$ & Social dimension & 0.637 & $* * *$ \\
\hline Entrepreneurial approach & $<-$ & Social dimension & 0.679 & $* * *$ \\
\hline Reporting compliance & $<-$ & Economic dimension & 0.488 & $* * *$ \\
\hline Revenue diversification & $<-$ & Economic dimension & 0.502 & $* * *$ \\
\hline Financial planning & $<-$ & Economic dimension & 0.778 & $* * *$ \\
\hline Stability of revenue & $<-$ & Economic dimension & 0.530 & $* * *$ \\
\hline
\end{tabular}

Similarly, the social dimension and economic dimension were found to be significant measures of Host organization sustainability. The highest value of the Host organization sustainability measure was evident in the economic dimension, with a path coefficient of 0.612 , while the social dimension had a path coefficient value of $0.368, p<0.001$. Therefore,

Hypothesis 2. Social dimension and economic dimension are measures of organizational sustainability.

is supported.

Finally, CbP Sustainability was found to significantly and positively influence Host organization sustainability. The path coefficient values showed that the CbP Sustainability significantly and positively influenced Host organization sustainability, showing a path coefficient value of $0.742,(p<0.001)$, which supports the research

Hypothesis 3. CbP sustainability positively and significantly influences Host organization sustainability.

In the literature, the relationship between the constructs had not been tested. The results of the SEM indicate that CbP Sustainability factors not only influence Host organization sustainability, but is also an antecedent of it.

The absolute fit indices for the model were good with a CMIN/DF of 1.545, GFI of 0.953, AGFI of 0.853, and CFI value in the model of 0.912. The RMSEA value in this model was 0.049 .

Therefore, all research hypotheses were confirmed. Table 10 summarizes the results. 
Table 10. Hypotheses accepted after data analysis.

\begin{tabular}{cccc}
\hline Hypotheses & Path Coefficient & Significance & Status \\
\hline $\begin{array}{c}\text { H1: Program specific, Organization specific and Community } \\
\text { specific factors are measures of CbP Sustainability }\end{array}$ & $0.703,0.682,0.533$, & $p<0.001$ & Supported \\
\hline $\begin{array}{c}\text { H2: Social dimension and Economic dimension are measures } \\
\text { of Host organization sustainability }\end{array}$ & $0.368,0.612$ & $p<0.001$ & Supported \\
\hline $\begin{array}{c}\text { H3: CbP Sustainability positively and significantly influences } \\
\text { Host organization sustainability }\end{array}$ & 0.742 & $p<0.001$ & Supported \\
\hline
\end{tabular}

\section{Conclusions}

The literature on healthcare community-based programs or initiatives is very diverse [103]. Most of the papers have described and analyzed specific factors for the success or continuity of CbPs [103-105].

The findings of this study bring new insights into the relationship between the sustainability of community-based programs and the sustainability of the host organizations, a field of research scarcely covered by the literature to date. One of the key premises of the research was that the three factors-program specific, organizational specific, and community specific—are significant for $\mathrm{CbP}$ Sustainability. This was found to be true.

Throughout this paper, healthcare was the setting and not the focus of the paper. In fact, it is very difficult to target broader types of healthcare $\mathrm{CbPs}$, since they are both very diverse (targeted community, local conditions, funding entities, stakeholders, and organizational setting to implement the program) and stakeholders may assess their results differently.

Another issue regards their sustainability. To make matters worse, no agreed-upon definition exists for the term healthcare program sustainability [106]. While in the case of organizations (both for and non-profit), these are mostly responsible for their long-term sustainability, CbPs in most cases rely more on external factors. Sometimes, sustainability is included as part of the implementation, narrowing the perspective in that way. Furthermore, factors that influence the successful initial implementation of $\mathrm{CbPs}$ are not necessarily the same factors that enable continued implementation. Healthcare community-based programs take place in both clinical and community settings, with interventions delivered by individual providers; other programs more often occur in a community setting such as community partnerships. This is why we had to consider, in our analysis, factors related to the host organizations, the programs themselves, and the communities where $\mathrm{CbPs}$ have been implemented. On the other hand, the sustainability factors had to be general, since each type of healthcare program has its own clinical setting.

In this research, considering all the factors, program specific was the most prominent measure of the CbP Sustainability construct with a path coefficient value of $0.703, p<0.001$, in the SEM model. The result indicates that the program specific is a key factor that enables $C b P$ Sustainability. $\mathrm{CbP}$ sustainability may be compromised without first considering the program itself. Program coordinator competence, program transparency, involvement of qualified staff in all stages of the program, the ability to address changes of community needs, making resources, and especially financial resources, available, a coherent framework, capability to document program success to make it visible for stakeholders, the ability of the program to adapt and evolve from the original plan, according to changing circumstances, and to align to the reporting requirements of stakeholders, accepting and integrating champions, have a profound integration with the host organization at all levels, having a congruent mission, capability to identify and integrate community needs and resources, but also stakeholder management by adapting to their policies and regulations, are elements that have to be considered by $\mathrm{CbP}$ initiators to increase the chances for success.

The organizational specific construct was found to influence $C b P$ Sustainability, with a path coefficient value of 0.682 and $p<0.001$. The host organization's senior management capacity to establish organizational goals congruent with the $\mathrm{CbP}$, its procedures, and mechanisms, the ability to adapt its internal regulations and procedures to various requirements, its capacity to initiate 
and maintain relations with multiple partners, but also specific organizational actions targeting sustainability may influence community-based program sustainability.

While various scholars consider the community more than other factors as critical for $\mathrm{CbP}$ sustainability, in this study, community specific variables, even though important, placed only third, with a path coefficient value of 0.16 and $p<0.001$. Although the results were significant, the path coefficient value was lower when compared to other dimensions, particularly those that were program specific. This is an indication that for many, the success and continuation of a community-based program relies more on the features of the program itself and the support of the host organization than the community where it is implemented. Since usually the communities targeted by CbPs are poor, this is understandable. Still, in designing an effective $\mathrm{CbP}$, elements like community involvement in $\mathrm{CbP}$ planning and implementation, community relations with various public or private bodies and agencies, and involvement in providing additional resources to $\mathrm{CbP}$, particularly financial contributions or target group(s) availability for $\mathrm{CbP}$ are important.

In terms of Host organizations sustainability, the economic dimension is more important than the social one ( 0.612 versus 0.368 ). The analysis of the findings also showed that CbP Sustainability factors positively impacted on the Host organization sustainability, which was measured through two dimensions: social and economic. The complete SEM model showed a path coefficient value of 0.742 with $p<0.001$, indicating significant impact of CbP Sustainability on Host organization sustainability. Since the entire conceptual model was studied as an input-output mode, it was hypothesized that $C b P$ Sustainability factors are input measures that impact on Host organization sustainability.

In terms of future research, there are several avenues which have to be explored: (1) A thorough analysis regarding the extent of each $\mathrm{CbP}$ Sustainability variable on specific factors of the host organization's sustainability has to be undertaken; and (2) the development of frameworks and conceptual models regarding the factors likely to affect the sustainability of healthcare. These frameworks have to cover all three major stakeholders of the CbPs: the community, funding provider, and host organization. This paper focused on host organizations and considered their perspective, while neglecting to some extent the community and fund providers. Perhaps combining frameworks developed from the perspective of all stakeholders will make the assessment simpler and consider healthcare CbPs. (3) Sustainability has to be analyzed both in terms of outcomes and processes. There seems to be, in the literature, two streams of healthcare CbPs sustainability research: one focused on the phases of community-based program operationalization that focuses on various sustainability issues during planning, implementation etc., while the second stream focuses on the outcomes, putting aside various phases in implementation.

Author Contributions: Conceptualization, S.I.C. and E.G.C.; Methodology, S.I.C. and E.G.C.; Validation, S.I.C. and E.G.C.; Formal analysis, S.I.C. and E.G.C.; Investigation, S.I.C. and E.G.C.; Writing-original draft preparation, S.I.C. and E.G.C.; Writing - review and editing, S.I.C. and E.G.C.; Funding acquisition, S.I.C.

Funding: This research was funded by PN-III-P4-ID-PCCF-2016-0084 Intelegerea si modelarea structurilor spatio-temporale ale inegalitatilor si polarizarii in relatie cu caracteristicile psihologice.

Conflicts of Interest: The authors declare no conflicts of interest.

\section{References}

1. Savaya, R.; Spiro, S.; Elran-Barak, R. Sustainability of social programs a comparative case study analysis. Am. J. Eval. 2008, 29, 478-493. [CrossRef]

2. Tomioka, M.; Braun, K.L. Examining sustainability factors for organizations that adopted Stanford's chronic disease self-management program. Front. Public Health 2014, 2, 158-165. [CrossRef] [PubMed]

3. Oino, P.G.; Towett, G.; Kirui, K.K.; Luvega, C. The Dilemma in Sustainability of Community-Based Projects in Kenya. Glob. J. Adv. Res. 2015, 2, 757-768.

4. Ceptureanu, S.I.; Ceptureanu, E.G.; Luchian, C.E.; Luchian, I. Community-based programs sustainability. A multidimensional approach. Sustainability 2018, 10, 870. [CrossRef] 
5. Argaw, D.; Fanthahun, M.; Berhane, Y. Sustainability and factors affecting the success of community-based reproductive health programs in rural Northwest Ethiopia. Afr. J. Reprod. Health 2007, 11, 79-88. [CrossRef]

6. Bennett, S.; Singh, S.; Ozawa, S.; Tran, N.; Kang, J.S. Sustainability of donor programs: Evaluating and informing the transition of a large HIV prevention program in India to local ownership. Glob. Health Action 2011, 4, 1-9. [CrossRef]

7. Estabrooks, P.A.; Smith-Ray, R.L.; Dzewaltowski, D.A.; Dowdy, D.; Lattimore, D.; Rheaume, C.; Wilcox, S. Sustainability of evidence-based community-based physical activity programs for older adults: Lessons from Active for Life. Transl. Behav. Med. 2011, 1, 208-215. [CrossRef]

8. Gatchell, V.; Forsythe, V.; Thomas, P.R. The sustainability of community-based therapeutic care (CTC) in nonemergency contexts. Food Nutr. Bull. 2005, 27,90-98. [CrossRef]

9. Hacker, K.; Tendulkar, S.A.; Rideout, C.; Bhuiya, N.; Trinh-Shevrin, C.; Savage, C.P.; DiGirolamo, A. Community capacity building and sustainability: Outcomes of community-based participatory research. Prog. Community Health Partnersh. 2012, 6, 349-360. [CrossRef]

10. Hanson, H.M.; Salmoni, A.W. Stakeholders' perceptions of programme sustainability: Findings from a community-based fall prevention programme. Public Health 2011, 125, 525-532. [CrossRef]

11. Mijnarends, D.; Pham, D.; Swaans, K.; Van Brakel, W.H.; Wright, P. Sustainability criteria for CBR programmestwo case studies of provincial programmes in Vietnam. Disabil. CBR Incl. Dev. 2011, 22, 3-21. [CrossRef]

12. Montemurro, G.R.; Raine, K.D.; Nykiforuk, C.I.; Mayan, M. Exploring the process of capacity-building among community-based health promotion workers in Alberta, Canada. Health Promot. Int. 2014, 29, 463-473. [CrossRef]

13. Gruen, R.L.; Elliott, J.H.; Nolan, M.L.; Lawton, P.D.; Parkhill, A.; McLaren, C.J.; Lavis, J.N. Sustainability science: An integrated approach for health-programme planning. Lancet 2008, 372, 1579-1589. [CrossRef]

14. Shediac-Rizkallah, M.C.; Bone, L.R. Planning for the sustainability of community-based health programs: Conceptual frameworks and future directions for research, practice and policy. Health Educ. Res. 1998, 13, 87-108. [CrossRef]

15. Sridharan, S.; Go, S.; Zinzow, H.; Gray, A.; Barrett, M.G. Analysis of strategic plans to assess planning for sustainability of comprehensive community initiatives. Eval. Program Plan. 2007, 30, 105-113. [CrossRef]

16. Cassidy, E.F.; Leviton, L.C.; Hunter, D.E.K. The relationship of program and organizational capacity to program sustainability. Eval. Program Plan. 2006, 29, 149-152. [CrossRef]

17. Sarriot, E.G.; Winch, P.J.; Ryan, L.J.; Edison, J.; Bowie, J.; Swedberg, E.; Welch, R. Qualitative research to make practical sense of sustainability in primary health care projects implemented by non-governmental organizations. Int. J. Health Plan. Manag. 2004, 19, 3-22. [CrossRef]

18. Scheirer, M.A. Is Sustainability Possible? A Review and Commentary on Empirical Studies of Program Sustainability. Am. J. Eval. 2005, 26, 320-347. [CrossRef]

19. Swerissen, H.; Crisp, B.R. The sustainability of health promotion interventions for different levels of social organization. Health Promot. Int. 2004, 19, 123-130. [CrossRef]

20. Glasgow, R.E.; Vogt, T.M.; Boles, S.M. Evaluating the public health impact of health promotion interventions: The RE-AIM framework. Am. J. Public Health 1999, 89, 1322-1327. [CrossRef]

21. Steckler, A.; Goodman, R.M. How to institutionalize health promotion programs. Am. J. Health Promot. 1989, 3, 34-43. [CrossRef] [PubMed]

22. DeMiglio, L.; Williams, A.M. A qualitative study examining the sustainability of shared care in the delivery of palliative care services in the community. BMC Palliat. Care 2013, 12, 32. [CrossRef] [PubMed]

23. Mancini, J.A.; Marek, L.I. Sustaining community-based programs for families. Fam. Relat. 2004, 53, $339-347$. [CrossRef]

24. Cleaver, F. Paradoxes of participation: Questioning participatory approaches to development. J. Int. Dev. 1999, 11, 597-612. [CrossRef]

25. Mulwa, F. Demystifying Participatory Community Development (rev. ed.); Pauline's Publications Africa: Nairobi, Kenya, 2010.

26. Cornwall, A.; Lucas, H.; Pasteur, K. Introduction: Accountability through participation: Developing workable partnership models in the health sector. IDS Bull. 2000, 31, 1-13. [CrossRef]

27. Matthews, J.; Herbert, D. (Eds.) Unifying Geography: Common Heritage, Shared Future? Oxfordshire: Routledge, UK, 2004. 
28. Pluye, P.; Potvin, L.; Denis, J. Making public health programs last: Conceptualizing sustainability. Eval. Program Plan. 2004, 27, 121-133. [CrossRef]

29. Sharir, M.; Lerner, M. Gauging the success of social ventures initiated by individual social entrepreneurs. J. World Bus. 2006, 41, 6-20. [CrossRef]

30. LaFond, A.K.; Brown, L.; Macintyre, K. Mapping capacity in the health sector: A conceptual framework. Int. J. Health Plan. Manag. 2002, 17, 3-22. [CrossRef] [PubMed]

31. Nu'Man, J.; King, W.; Bhalakia, A.; Criss, S. A framework for building organizational capacity integrating planning, monitoring, and evaluation. J. Public Health Manag. Pract. 2007, 13, S24-S32. [CrossRef]

32. Jacobs, B.; Price, N.; Sam, S.O. A sustainability assessment of a health equity fund initiative in Cambodia. Int. J. Health Plan. Manag. 2007, 22, 183-203. [CrossRef]

33. Sarriot, E.G.; Winch, P.J.; Ryan, J.; Bowie, J.; Kouletio, M.; Swedberg, E.; LeBan, K.; Edison, J.; Welch, R.; Pacqué, C. A methodological approach and framework for sustainability assessment in NGO-implemented primary health care programs. Int. J. Health Plan. Manag. 2004, 19, 23-41. [CrossRef] [PubMed]

34. Light, P.C. Sustaining Innovation: Creating Nonprofit and Government Organizations that Innovate Naturally; Jossey-Bass: San Francisco, CA, USA, 1998.

35. Steadman, H.J.; Cocozza, J.J.; Dennis, D.L.; Lassiter, M.G.; Randolph, F.L.; Goldman, H.; Blasinsky, M. Successful program maintenance when federal demonstration dollars stop: The access program for homeless mentally ill persons. Adm. Policy Ment. Health 2002, 29, 481-493. [CrossRef] [PubMed]

36. Padgett, S.M.; Bekemeier, B.; Berkowitz, B. Building sustainable public health systems change at the state level. J. Public Health Manag. Pract. 2005, 11, 109-115. [CrossRef] [PubMed]

37. Weiss, H.; Coffman, J.; Bohan-Baker, M. Evaluation's Role in Supporting Initiative Sustainability. In Proceedings of the Fifth Bi-Annual Meeting of the Urban Seminar Series on Children's Health and Safety at Harvard University, Cambridge, MA, USA, 5-6 December 2002.

38. Akerlund, K.M. Prevention program sustainability: The state's perspective. J. Community Psychol. 2000, 28, 353-362. [CrossRef]

39. O'Loughlin, J.; Renaud, L.; Richard, L.; Gomez, L.S.; Paradis, G. Correlates of the sustainability of community-based heart health promotion intervention. Prev. Med. 1998, 27, 702-712. [CrossRef]

40. Holder, H.D.; Moore, R.S. Institutionalization of community action projects to reduce alcohol use and related problems: Systematic facilitators. Subst. Use Misuse 2000, 35, 75-86. [CrossRef]

41. Goodson, P.; Smith, M.M.; Evans, A.; Meyer, B.; Gottlieb, N.H. Maintaining prevention in practice: Survival of PPIP in primary care settings. Am. J. Prev. Med. 2001, 20, 184-189. [CrossRef]

42. Stevens, B.; Peikes, D. When the funding stops: Do grantees of the Local Initiative Fund Partners Program sustain themselves? Eval. Program Plan. 2006, 29, 153-161. [CrossRef]

43. Pentz, M.A. Institutionalizing community-based prevention through policy change. J. Community Psychol. 2000, 28, 257-270. [CrossRef]

44. Johnson, K.; Hays, C.; Center, H.; Daley, C. Building capacity and sustainable prevention innovations: A sustainability planning model. Eval. Program Plan. 2004, 27, 135-149. [CrossRef]

45. Savaya, R.; Elsworth, G.; Rogers, P. Projected sustainability of innovative social programs. Eval. Rev. 2008, 33, 189-205. [CrossRef] [PubMed]

46. Smith, M.; Buckwalter, K.C.; Zevenbergen, P.W.; Kudart, P.; Springer-Brenneman, D.; Garand, L. An administrator's dilemma: Keeping the innovative mental health and aging programs alive after the grant funds end. J. Ment. Health Adm. 1993, 20, 212-222. [CrossRef] [PubMed]

47. Mancini, J.A.; Martin, J.A.; Bowen, G.L. Community capacity. In Encyclopedia of Primary Prevention and Health Promotion; Gullotta, T.P., Bloom., M., Eds.; Kluwer Academic/Plenum: New York, NY, USA, 2003; pp. 319-330.

48. Beery, W.L.; Senter, S.; Cheadle, A.; Greenwald, H.P.; Pearson, D.; Brousseau, R.; Nelson, G. Evaluation of the legacy of community health initiatives. Am. J. Eval. 2005, 26, 150-165. [CrossRef]

49. Robinson, K.; Elliot, S.J.; Driedger, M.; Eyles, J.; O’Loughlin, J.; Riley, B.; Cameron, R.; Harvey, D.; CHHDP Strategic and Research Advisory Groups. Using linking systems to build capacity and enhance dissemination in heart health promotion: A Canadian multiple-case study. Health Educ. Res. 2005, 20, 499-513. [CrossRef]

50. Pluye, P.; Potvin, L.; Denis, J.; Pelletier, J.; Mannoni, C. Program sustainability begins with the first events. Eval. Program Plan. 2005, 28, 123-137. [CrossRef]

51. Weerawardena, J.; McDonald, R.E.; Sullivan Mort, G. Sustainability of non-profit organizations: An empirical investigation. J. World Bus. 2010, 45, 346-356. [CrossRef] 
52. Chetkovich, C.; Frumkin, P. Balancing margin and mission: Non-profit competition in charitable versus fee-based programs. Adm. Soc. 2003, 35, 564-596. [CrossRef]

53. McDonald, R.E. An investigation of innovation in non-profit organizations: The role of organizational mission. Non-Profit Volunt. Sect. Q. 2007, 36, 256-281.

54. Valentinov, V. The economics of non-profit organization: In search of an integrative theory. J. Econ. Issues 2008, 17, 745-761. [CrossRef]

55. Wijkstrom, F. The Swedish non-profit sector in international comparison. Ann. Public Coop. Econ. 1997, 68, 625-663. [CrossRef]

56. Dart, R. Being 'business-like' in a non-profit organization: A grounded and inductive typology. Non-Profit Volunt. Sect. Q. 2004, 33, 290-310. [CrossRef]

57. Goerke, J. Taking the quantum leap: Non-profits are now in business. An Australian perspective. Int. J. Non-Profit Volunt. Sect. Mark. 2003, 8, 317-327. [CrossRef]

58. Lee, L.; Piliavin, J.A.; Call, V.R.A. Giving time, money, and blood: Similarities and differences. Soc. Psychol. Q. 1999, 62, 276-290. [CrossRef]

59. Berger, I.E.; Cunningham, P.H.; Drumwright, M.E. Social alliances: Company/ non-profit collaboration. Calif. Manag. Rev. 2004, 47, 58-90. [CrossRef]

60. Handy, F.; Srinivasan, N. Improving quality while reducing costs? An economic evaluation of the net benefits of hospital volunteers. Non-Profit Volunt. Sect. Q. 2004, 33, 28-54. [CrossRef]

61. Snavely, K.; Tracy, M.B. Collaboration among rural non-profit organizations. Non-Profit Manag. Leadersh. 2000, 11, 145-165. [CrossRef]

62. Greenlee, J.S.; Trussel, J.M. Predicting the financial vulnerability of charitable organizations. Non-Profit Manag. Leadersh. 2000, 11, 199-210. [CrossRef]

63. Keating, E.K.; Fisher, M.; Gordon, T.P.; Greenlee, J.S. Assessing Financial Vulnerability in the Non-Profit Sector; KSG Faculty Research Working Paper Series RWP05-002; Hauser Center for Nonprofit Organizations Paper No. 27; Harvard University: Cambridge, MA, USA, 2005.

64. Prentice, C.R. Why So Many Measures of Non-profit Financial Performance? Analyzing and Improving the Use of Financial Measures in Non-profit Research. Non-Profit Volunt. Sect. Q. 2016, 45, 715-740. [CrossRef]

65. Weikart, L.; Chen, G.; Sermier, E. Budgeting E Financial Management for Non-Profit Organizations: Using Money to Drive Mission Success; CQ Press: Los Angeles, CA, USA, 2013.

66. McLaughlin, T. Streetsmart Financial Basics for Non-Profit Managers; John Wiley: Hoboken, NJ, USA, 2009.

67. Coe, C. Non-Profit Financial Management: A Practical Guide; John Wiley: Hoboken, NJ, USA, 2011.

68. Zietlow, J.; Hankin, J.; Seidner, A. Financial Management for Non-Profit Organizations: Policies and Practices; John Wiley: Hoboken, NJ, USA, 2007.

69. Okorley, E.L.; Nkrumah, E.E. Organizational factors influencing sustainability of local non-governmental organizations. Lessons from a Ghanaian context. Int. J. Soc. Econ. 2012, 39, 330-341. [CrossRef]

70. Schaffer, S. Success Factors for Non-profit Organizations. A New Approach to the Development of Thriving Mission-Driven Enterprises; Public Interest Management Group: Seattle, WA, USA, 2015.

71. Ceptureanu, S.I.; Ceptureanu, E.G.; Bogdan, V.L.; Radulescu, V. Sustainability Perceptions in Romanian Non-Profit Organizations: An Exploratory Study Using Success Factor Analysis. Sustainability 2018, 10, 294. [CrossRef]

72. Peredo, A.M.; McLean, M. Social entrepreneurship: A critical review of the concept. J. World Bus. 2006, 41, 56-65. [CrossRef]

73. Anderson, B.B.; Dees, J.G. Developing viable earned income strategies. In Strategic Tools for Social Entrepreneurs: Enhancing the Performance of Your Enterprising Non-Profit; Dees, J.G., Emerson, J., Economy, P., Eds.; John Wiley \& Sons, Inc.: New York, NY, USA, 2002; pp. 191-233.

74. Dees, J.G.; Emerson, J.; Economy, P. (Eds.) Strategic Tools for Social Entrepreneurs: Enhancing the Performance of Your Enterprising Non-Profit; John Wiley \& Sons, Inc.: New York, NY, USA, 2004.

75. Mair, J.; Marti, I. Social entrepreneurship research: A source of explanation, prediction and delight. J. World Bus. 2006, 41, 36-44. [CrossRef]

76. Eikenberry, A.M.; Kluver, J.D. The marketization of the non-profit sector: Civil society at risk. Public Adm. Rev. 2004, 64, 132-140. [CrossRef]

77. Nicholls, A. Social Entrepreneurship: New Models of Sustainable Social Change; Oxford University Press: Oxford, UK, 2006. 
78. Forehand, A. Mission and organizational performance in the healthcare industry. J. Healthc. Manag. 2000, 45, 267-277. [CrossRef]

79. Durst, S.L.; Newell, C. The who, why, and how of reinvention in non-profit organizations. Non-Profit Manag. Leadersh. 2001, 11, 443-457. [CrossRef]

80. Ceptureanu, S.I.; Ceptureanu, E.G.; Orzan, M.C.; Marin, I. Toward a Romanian NPOs Sustainability Model: Determinants of Sustainability. Sustainability 2017, 9, 966. [CrossRef]

81. Prahalad, C.K. The Fortune at the Bottom of the Pyramid: Eradicating Poverty through Profits; Wharton School Publishing: Upper Saddle River, NJ, USA, 2004.

82. Gray, B.; Stites, J.P. Sustainability through Partnerships. Capitalizing on Collaboration. Available online: http: //www.mspguide.org/resource/sustainability-through-partnerships-capitalizing-collaboration (accessed on 17 July 2019).

83. Helmig, B.; Jegers, M.; Lapsley, I. Challenges in Managing Non-profit Organizations: A Research Overview. Voluntas 2004, 15, 101-116. [CrossRef]

84. Thompson, J.L.; Alvy, G.; Lees, A. Social entrepreneurship-A new look at the people and the potential. Manag. Decis. 2000, 38, 328-338. [CrossRef]

85. Austin, J.; Stevenson, H.; Wei-Skillern, J. Social and commercial entrepreneurship: Same, different or both? Entrep. Theory Pract. 2006, 30, 1-22. [CrossRef]

86. Alvord, S.; Brown, L.; Letts, C. Social entrepreneurship \& societal transformation: An exploratory study. J. Appl. Behav. Sci. 2004, 40, 260-282.

87. Turner, D.; Martin, S. Social entrepreneurship and social inclusion: Building local capacity or delivering national priorities. Int. J. Public Adm. 2005, 28, 797-806. [CrossRef]

88. Parsons, E.; Broadbridge, A. Managing Change in Non-profit Organizations: Insights from the UK Charity Retail Sector. Voluntas 2004, 15, 227-242. [CrossRef]

89. Iwu, C.G.; Kapondoro, L.; Twum-Darko, M.; Tengeh, R. Determinants of Sustainability and Organizational Effectiveness in Non-Profit Organizations. Sustainability 2015, 7, 9560-9573. [CrossRef]

90. Murtaza, N. Putting the Lasts First: The Case for Community-Focused and Peer-Managed NGO Accountability Mechanisms. Voluntas 2012, 23, 109-125. [CrossRef]

91. Hodge, M.M.; Piccolo, R.F. Funding source, board involvement techniques, and financial vulnerability in non-profit organizations: A test of resource dependence. Non-Profit Manag. Leadersh. 2005, 16, 171-190. [CrossRef]

92. Hair, J.F.; Black, W.C.; Babin, B.J.; Anderson, R.E. Multivariate Data Analysis: A Global Perspective, 7th ed.; Pearson Education: Hoboken, NJ, USA, 2010.

93. Tabachnik, B.G.; Fidell, L.S. Using Multivariate Statistics; Pearson International Education: London, UK, 2007.

94. Lubke, G.; Muthen, H.; Bengt, O. Applying Multi-Group Confirmatory Factor Models for Continuous Outcomes to Likert Scale Data Complicates Meaningful Group Comparisons. Struct. Equ. Modeling 2004, 11, 514-534. [CrossRef]

95. Levine, T.R. Confirmatory factor analysis and scale validation in communication research. Commun. Res. Rep. 2005, 22, 335-338. [CrossRef]

96. Kline, R.B. Principles and Practice of Structural Equation Modeling, 3rd ed.; Guilford Press: New York, NY, USA, 2011.

97. Thompson, B. Exploratory and Confirmatory Factor Analysis: Understanding Concepts and Applications; American Psychological Association: Washington, DC, USA, 2004.

98. Pallant, J. SPSS Survival Manual, 3rd ed.; Open University Press: Berkshire, UK, 2005.

99. Byrne, B.M. Structural Equation Modeling with AMOS. Basics Concepts, Applications, and Programming; Lawrence Erlbaum Associates: Hillsdale, NJ, USA, 2006.

100. O'Brien, R.M. A Caution Regarding Rules of Thumb for Variance Inflation Factors. Qual. Quant. 2007, 41, 673-690. [CrossRef]

101. Mohsen, T.; Dennick, R. Making sense of Cronbach's Alpha. Int. J. Med Educ. 2011, 2, 53-55.

102. Russell, D.W. In search of underlying dimensions: The use (and abuse) of factor analysis in Personality and Social Psychology Bulletin. Personal. Soc. Psychol. Bull. 2002, 8, 1629-1646. [CrossRef] 
103. Sulistyawati, S.; Dwi Astuti, F.D.; Umniyati, S.R.; Satoto, T.B.T.; Lazuardi, L.; Nilsson, M.; Rocklov, J.; Andersson, C.; Holmner, A. Dengue Vector Control through Community Empowerment: Lessons Learned from a Community-Based Study in Yogyakarta, Indonesia. Int. J. Environ. Res. Public Health 2019, 16, 1013. [CrossRef] [PubMed]

104. Miltenburg, A.S.; van Pelt, S.; de Bruin, W.; Shields-Zeeman, L. Mobilizing community action to improve maternal health in a rural district in Tanzania: Lessons learned from two years of community group activities. Glob. Health Action 2019, 12, 1621590. [CrossRef] [PubMed]

105. Srivarathan, A.; Jensen, A.N.; Kristiansen, M. Community-based interventions to enhance healthy aging in disadvantaged areas: Perceptions of older adults and health care professionals. BMC Health Serv. Res. 2019, 19, 7. [CrossRef] [PubMed]

106. Scheirer, M.A. Linking Sustainability Research to Intervention Types. Fram. Health Matters 2013, 103, 73-80. [CrossRef]

(C) 2019 by the authors. Licensee MDPI, Basel, Switzerland. This article is an open access article distributed under the terms and conditions of the Creative Commons Attribution (CC BY) license (http://creativecommons.org/licenses/by/4.0/). 